\title{
Concreto ou abstrato? Um esquema conceitual das representações mentais da economia compartilhada
}

\section{Abstract or concrete? A conceptual framework of Sharing Economy's Construal Levels}

\author{
Jefferson Sordi \\ Universidade Feevale - Brasil \\ jefferson@feevale.br \\ ORCID: 0000-0001-5807-3796 \\ Maira Petrini \\ Pontifícia Universidade Católica do Rio Grande do Sul - PUCRS - Brasil \\ maira.petrini@pucrs.br \\ ORCID: 0000-0002-3914-2589 \\ Claudio Hofmann Sampaio \\ Pontifícia Universidade Católica do Rio Grande do Sul - PUCRS - Brasil \\ csampaio@pucrs.br \\ ORCID: 0000-0001-9943-1414
}

Submetido em 08/10/2018; Aprovado em 20/11/2018

\section{Resumo}

Objetivo: Discutir sobre as dimensões da economia compartilhada e como suas representações mentais influenciam o gap entre atitude e comportamento, tendo como base os arquétipos da economia compartilhada.

Metodologia/abordagem: Teórica e Conceitual

Principais resultados: A partir da análise teórica, propõe-se um esquema conceitual demonstrando que as dimensões representadas de forma mais abstrata podem impactar mais intensamente a atitude do consumidor sobre a economia compartilhada. Outras três dimensões, estas mais concretas, tendem a influenciar o comportamento com mais intensidade. Especula-se, também, que o modelo de negócio da economia compartilhada também pode sofrer influências dos níveis de representação mental.

Contribuições teóricas/metodológicas: 0 artigo contribui à literatura ao sugerir que mecanismos psicológicos dos indivíduos possuem influência na participação na economia compartilhada. Ao propor que o gap pode ter intensidade variada pelo tipo de negócio, respaldado pelos arquétipos de Petrini et al. (2017), o artigo tenta expandir o modelo de Hamari et al. (2015) e trazer explicações alternativas para sua ocorrência.

Relevância/originalidade: Este é um dos primeiros trabalhos que utilizam os níveis de interpretação mental como possível explicação para o acesso à economia compartilhada. Além disso, percebeu-se que a literatura apresenta uma série de trabalhos dispostos a discutir os motivos de participar no consumo colaborativo. Entretanto, são poucas as abordagens desse fenômeno pelo viés da psicologia, através da Construal Level Theory.

Palavras-Chave: Economia compartilhada; Consumo Colaborativo; Construal Level Theory;

\footnotetext{
Abastract

Objective: Discuss the dimensions of sharing economy and how their construal levels influence the gap between attitude and behavior, based on the archetypes of sharing economy.

Method: Theorical and Conceptual

Results: From the theoretical analysis, a conceptual scheme is proposed, demonstrating that the dimensions represented in a more abstract way can impact more intensely the attitude of the consumer on the shared economy. Three other dimensions, these more concrete, tend to influence behavior with more intensity. It is also speculated that the business model of the shared economy can also be influenced by mental representation levels.
} 
Theoretical contributions: The article contributes to the literature by suggesting that individuals' psychological mechanisms influence participation in the shared economy. By proposing that the gap can be varied according to the type of business, supported by the archetypes of Petrini et al. (2017), the article attempts to expand the model of Hamari et al. (2015) and bring alternative explanations for the phenomenon.

Relevance: This is one of the earliest works that use levels of mental interpretation as a possible explanation for access to the shared economy. In addition, it was noticed that the literature presents a series of works willing to discuss the reasons to participate in the collaborative consumption. However, there are few approaches to this phenomenon from the bias of psychology, through Construal Level Theory.

Keywords: Sharing Economy; Collaborative Consumption Construal Level Theory;

\section{Introdução}

A economia compartilhada, também chamada de consumo colaborativo de forma intercambiável (Martin, 2016), é um movimento contemporâneo que reflete uma resposta da sociedade e das organizações à busca pelo desenvolvimento sustentável, trazendo consigo uma infinidade de desafios que precisam ser superados. Tal movimento é expresso em novas formas e modelos de compartilhamento e acesso a bens ou serviços. 0 compartilhamento constitui-se em uma atividade presente em todas as eras da civilização (Price \& Belk, 2016), seja através da distribuição de comida entre os familiares e amigos em festas, seja através de conversas ou qualquer outra forma de estabelecer laços entre pessoas e grupos (Godelier, 2012). 0 fenômeno do consumo colaborativo fora explorado academicamente há pelo menos quarenta anos, quando Felson e Spaeth (1978) publicaram seu trabalho. Baseados em situações que as pessoas se juntavam para consumir determinados produtos. Então, sendo assim, o que há de contemporâneo neste movimento? A escala que ele toma, uma vez que esse compartilhamento, antes restrito a família, amigos ou pequenos grupos, passa a ser realizado entre estranhos, dada a evolução das plataformas tecnológicas. Com isso, a expressão "economia compartilhada" tem-se feito presente nos últimos tempos nas discussões de modelos de negócios (Botsman \& Rogers, 2011), pois as práticas comerciais (que envolvam a troca monetária, ou não) fazem parte de uma nova onda econômica que surgiu (Rudmin, 2016).

No contexto brasileiro, algumas pesquisas começam a mostrar alguns resultados sobre a participação da população na economia compartilhada. Markus e Orsi (2016) comentam que ainda existe de uma falta de estrutura, em especial tecnológica, para uma adesão mais contundente por parte dos brasileiros à economia compartilhada. Esse resultado encontra eco no trabalho de Piran, Neves, Sordi e Nunes (2018) que destaca, além desse ponto de estrutura, que há um interesse pelo tema, também, pela possibilidade de geração de novos negócios, cuidando o que Branco e Nunes (2018) identificaram como a possível precarização do trabalho nessas plataformas.

Na busca por melhor delimitar os modelos de negócio que emergem sob o guarda-chuva desta "nova" economia, Habibi, Kim e Laroche (2016) desenvolveram um framework, fundamentado nas características de protótipo de Belk (2010), que mensura e posiciona os tipos de negócios dentro de um continuum, a partir do cálculo do seu “Índice de Compartilhamento (Sharing Score). É possível observar a existência de negócios de puro compartilhamento, onde elementos como ligações sociais e ausência de transação monetária são mais presentes, e negócios de pura troca, onde o dinheiro, a reciprocidade e a troca balanceada são pontos essenciais para seu funcionamento (Habibi, Davidson \& Laroche, 2017). Em uma proposta mais classificatória, Petrini, Freitas e Silveira (2017) identificam três arquétipos: New Business Models, Redesigned Businesses e Sharing by Ideal, nos quais os diferentes modelos de economia compartilhada e consumo colaborativo podem ser classificadas. Os arquétipos são propostos a partir da análise de quatro características identificadas em modelos de economia compartilhada: tipo de plataforma, transação financeira, estrutura de mercado e modelo de compartilhamento. 0 tipo de plataforma pode ser interferente ou intermediadoras, envolvendo ou não a presença de transações financeiras. A estrutura de mercado relaciona-se à propriedade do bem que está sendo compartilhado, se indivíduos ou organizações. Por fim, o modelo de compartilhamento pode se dar baseado no acesso ao bem ou na transferência da propriedade deste bem.

A literatura e as pesquisas acadêmicas já apresentam algumas discussões sobre o que leva um consumidor a participar desse tipo de consumo colaborativo. Uma parte desse campo, destina-se a 
entender as dimensões que estão por trás do consumo colaborativo. Nesse contexto, é possível destacar os trabalhos de Lamberton e Rose (2012), Pizzol e Almeida (2015), Mölmann (2015) e Hamari; Sjöklint e Ukkonen (2015). Os quatro trabalhos investigam o consumo colaborativo analisando modelos de negócio baseados no acesso. É o exemplo dos alugueis por curto espaço de tempo, como AirBnb e ZipCar, duas plataformas que se destinam a conectar ofertantes e demandantes de espaço ou transporte, respectivamente. Piscicelli, Cooper e Fischer (2015) e Lawson, Gleim, Perren e Hwang (2016) buscaram compreender como os valores pessoais (Schawartz et al. 2001; 2012) poderiam afetar a aceitação, adoção e difusão do consumo colaborativo. Com o intuito de entender que pontos mais impactavam a atitude e o comportamento (Ajzen, 1991), Hamari et al. (2015) identificam um gap importante para a literatura de economia compartilhada. Os autores sustentam que existe uma discrepância entre as atitudes e opiniões das pessoas sobre a economia compartilhada e sua efetiva participação nela. Ou seja, as pessoas enxergam os modelos do consumo colaborativo como positivos, mas aderem em menor intensidade a eles. Essa análise é feita a partir dos impactos das dimensões propostas pelos autores nos construtos da atitude e do comportamento.

Uma possível explicação para esse gap pouco trabalhado pela literatura, seja de comportamento do consumidor, seja de economia compartilhada, pode ser dada a partir da influência dos níveis de interpretação mental de cada indivíduo sobre suas decisões de consumo. Essa é a essência da Construal Level Theory (CLT) de Trope e Libermann (2003). Para Reyt e Wiesenfeld (2015) qualquer objeto ou situação pode ser estudado à luz da CLT. A CLT trata da existência de distâncias psicológicas para os seres humanos, assumindo que suas reações a eventos psicologicamente distantes e próximos possuem diferenças. A principal distinção é que os eventos mais distantes são interpretados em altos níveis, onde a abstração está mais presente, enquanto para eventos ou objetos mais próximos, essa interpretação é feita de forma mais concreta e, portanto, de baixo nível. A possível existência de relação entre os níveis de interpretação mental e o consumo colaborativo delimita a questão de pesquisa deste artigo: como podem ser construídas as dimensões do consumo colaborativo na mente do consumidor?

Esse ensaio se propõe a discutir sobre as dimensões do consumo colaborativo e como suas representações podem influenciar o gap entre atitude e comportamento, observado por Hamari et al. (2015). Uma vez que o consumo colaborativo se manifesta de formas bastante diversas, pretende-se compreender, ainda, como a participação nos diferentes arquétipos (Petrini et al., 2017), pode ser afetada pela representação mental dessas dimensões. Para alcançar esses objetivos, o presente artigo revisita a literatura sobre os níveis de construção mental e sobre a economia compartilhada em suas dimensões e tipologias. Por fim, a partir de uma discussão sobre os temas, propõe-se um esquema teórico que relaciona as dimensões do consumo colaborativo e o gap entre atitude e comportamento.

\section{Níveis de Interpretação Mental}

Os níveis de representação mental das pessoas informam como as mesmas classificam um objeto, evento ou indivíduo, a partir da distância psicológica existente (Trope \& Liberman, 2003). Essa premissa está presente na Construal Level Theory, que deriva da Psicologia Social. A distância psicológica é entendida como a sensação de que o ponto analisado está afastado da experiência presente e do ego do indivíduo (Trope \& Liberman, 2010). 0 ser humano consegue construir essas representações mentais e através delas reflete sobre o fato de estar distante, ou próximos, de objetos, pessoas ou eventos. Essas representações podem ser de nível alto, que envolvem considerações mais abstratas, sem tantos detalhes, ou de nível mais baixo, concretas e detalhadas (Trope \& Liberman, 2003; 2010).

As distâncias psicológicas são trabalhadas em quatro tipos: social, hipotética, temporal e geográfica - essa última também chamada de espacial (Liberman \&Trope, 2014). A distância social, por exemplo, é trabalhada a partir de avaliações de um comportamento realizados para si (próximo) ou para outra pessoa (distante) (Zao \& Xie, 2011). Uma distância hipotética avalia a possibilidade de uma ocorrência na vida de um ser humano. Quanto maior a probabilidade, mais próxima - e concreta - está do indivíduo (Lamberton \& Diehl, 2013). A distância geográfica envolve julgamentos sobre ações espacialmente próximas ou distantes do consumidor (Goodman \& Malkoc, 2012). A distância temporal envolve a observação de eventos próximos ou distantes no horizonte temporal do indivíduo. Foi a distância primordial de Trope e Liberman (2003) em seu trabalho seminal.

Além desses pontos, a CLT também é pesquisada através de duas formas de variação no mindset do indivíduo. Primeiro, pelas características de determinado objeto de avaliação. Ao se colocar ênfase nas questões primárias do objeto analisado, aquelas ligadas a sua finalidade, se ativa representações 
mentais abstratas. Em contraste, ao recair o foco sobre questões secundárias, são ativadas representações mais concretas (Liberman \& Trope; 2014; Irmak, Wakslak \& Trope, 2013; Chen, Shu \& Shen, 2017). Segundo, pelo condicionamento direto do respondente em pensar de forma concreta ou abstrata. Ao pedir que a pessoa se concentre em como (how) realizar determinada ação, se está ativando um nível mais concreto de construção mental (Trope \& Liberman, 2003). Em contrapartida, ao levar a atenção para os motivos do porquê (why) seguir determinada atividade, é possível iniciar um nível abstrato na mente do indivíduo (Cheema \& Patrick, 2008; Tsai \& McGill, 2011; Yan \& Sengupta, 2011; Cho, Khan \& Dhar, 2013)

A CLT pode ser aplicada a diversos contextos. Como explicam Reyt e Wiesenfeld (2015), a teoria consegue ajustar-se a um determinado domínio e ser estuda especificamente para essa situação. Isso permite sua aplicação aos campos psicológico (Trope \& Liberman, 2003), organizacional (Wiesenfeld, Reyt, Brockner \& Trope, 2017) e de marketing, em especial ao comportamento do consumidor (Trope, Liberman \& Wakslak, 2007).

\section{Economia Compartilhada}

Botsman e Rogers (2011) denominam como economia compartilhada os modelos comerciais que fazem com que bens e serviços possam ser acessados por consumidores, mesmo que não haja, necessariamente, a compra ou a troca de valores entre as partes envolvidas. Uma outra forma de designar esse fenômeno é defendida por Dubois, Shor e Carfagna (2014), que o chamam de connected consumption (consumo conectado), onde as práticas de reutilização de produtos e conexões peer-to-peer (entre pares) eliminam intermediários. Essa situação tem poder suficiente para modificar a percepção das pessoas junto às organizações, criando novos modelos de negócios, focados no compartilhamento (Gansky, 2010).

Antes de avançar na temática, é importante e necessário alguns esclarecimentos sobre o compartilhamento. Rudmin (2016, p.198) define o ato de compartilhar (sharing) como "o uso simultâneo ou sequencial de bens (carros, livro, comida, água), espaços (salas de estar, jardins, websites) ou intangibilidades (experiências, crenças, identidades, hereditariedade) por mais de um indivíduo". Outra definição importante para esse momento é a de Belk (2007, p. 26). Para o autor o compartilhamento é "o ato e processo de distribuir o que é seu para outras pessoas usarem e/ou o ato ou processo de receber ou pegar algo de outros para o próprio uso". Ficam claras nessas duas ideias que o compartilhamento envolve o ato de colocar à disposição para uso de terceiros alguns itens de sua propriedade. Rudmin (2016) complementa, sustentando que se existe a possibilidade de um recurso ser partilhado por múltiplos usuários, o compartilhamento acontece naquela situação. E isso ocorre quando os donos decidem compartilhar suas propriedades, ou porque o item é coletivo em sua forma inicial de propriedade.

Em essência, apesar de ser considerado um movimento novo e revolucionário, Rudiman (2016) salienta que o compartilhamento acontece há muitos séculos. Gollnhoffer, Hellwig e Morhart (2016) salientam que o comportamento de compartilhar começa a partir das esferas domésticas e transferemse para pontos mais distantes socialmente. Nesse caminho, o surgimento de organizações que destinamse a estabelecer pontes entre participantes do sistema de consumo colaborativo, torna-se um modelo alternativo para conseguir determinados itens (Sun, Supangkat \& Balarubramanian, 2016).

O surgimento da economia compartilhada parece ter suas raízes em três elementos que ocorrem conjuntamente. 0 primeiro deles é o fator tecnológico, uma vez que essas conexões de desconhecidos acontecem em plataformas online e necessitam de grandes avanços em desenvolvimento de máquinas, equipamentos e softwares (Gansky, 2010; Kaplan \& Haenlein, 2010; Schor, 2014). 0 segundo deles reside na discussão do acesso em detrimento à propriedade (Richins, 1994; Rifkin, 2000; Chen 2009; Bardhi \& Eckhardt, 2012). Acesso e partilha, analisados a luz da propriedade, são similares uma vez que em ambos o consumo não envolve transferência de propriedade, entretanto eles diferem no que diz respeito ao senso de propriedade percebida. Esse cenário leva à ideia de que a posse dos produtos não é mais tão vantajosa, pois não é necessário tê-los para usufruir dos benefícios que eles entregam, além de aumentar a gama de opções de produtos e serviços a que se tem acesso (Wolcott, 2014). Por fim, o terceiro elemento pauta-se na transição para uma sociedade mais sustentável, na qual experimentamse novas formas de satisfazer as exigências dos consumidores buscando menos prejuízos ao meio ambiente. Belk (2014) destaca que fatores como o aquecimento global, aumento dos preços dos combustíveis e matérias-primas e aumento da poluição, também estimulam oportunidades para o 
compartilhamento. Nesse mesmo sentido, e também como uma resposta potencial ao hiperconsumo, muitos consumidores se voltam para formas alternativas de consumo sustentável (Schor, 2014; Mölmann,2015).

0 trabalho de Hamari et al. (2015) sugere a existência de um gap entre a atitude e o comportamento dentro da economia compartilhada. Ou seja, as pessoas percebem positivamente e expressam opiniões positivas sobre a economia compartilhada, mas isso não necessariamente se transforma em ação. Apesar da relação significativa entre atitude e comportamento, ao mesma não possui intensidade semelhante a estudos do mesmo estilo. É o que Arts, Frambach e Bijmolt (2011), de forma irônica, comentam ser o efeito de respostas que os entrevistados de surveys dão por acharem ser as melhores. Os autores falam em um "talk the talk" que não se converte em um "walk the walk" quando se está falando de adoção de inovações. 0 que se quer, portanto, com o presente artigo, é propor uma explicação para o acontecimento desse gap, a partir das interpretações mentais das dimensões e dos tipos de economia compartilhada.

\subsection{Dimensões da Economia Compartilhada}

Com o intuito de usar as ideias da CLT para explicar o processo de participação na economia compartilhada, é importante ter compreensão acerca das dimensões do consumo colaborativo, exploradas por Lamberton e Rose (2012), Pizzol e Almeida (2015), Hamari et al. (2015) e Mohlmann (2015). A partir delas, se discutirá como as mesmas podem ser representadas mentalmente e como isso afeta, em última instância, a interpretação mental dos tipos de negócio e seus efeitos na atitude e no comportamento. A escolha por esses estudos se dá pelo fato de que todos tentam apresentar elementos que possuem potencial de afetar a participação no contexto do consumo colaborativo, mesmo que o texto de Hamari et al. (2015) tenha se dedicado a investigar a formação de atitudes e comportamentos, enquanto Pizzol e Almeida (2015) tenham optado por analisar o construto em si, por exemplo.

0 número de dimensões formadoras do consumo colaborativo, bem como suas nomenclaturas mudam de trabalho para trabalho. Entretanto, seus significados não variam tanto. Por exemplo, o que Lamberton e Rose (2015) denominam "Familiaridade" é associável ao que Pizzol e Almeida (2015) identificam como "Riscos". Em ambos os casos, a dimensão está associada ao conhecimento sobre o funcionamento dos negócios dentro do ambiente do consumo colaborativo. Hamari et al. (2015) apresentam sua dimensão de "Benefícios Econômicos" com um sentido semelhante à "Economia de Custos" de Mölmann (2015), com os dois textos comprovando que os valores economizados com esse tipo de transação estão presentes. Pizzol e Almeida (2015), Hamari et al. (2015) e Mölmann (2015) adotam três nomes distintos para tratar da importância de aspectos sócio-ambientais para essa nova onda econômica: "Consciência Sócio-Ambiental", "Sustentabilidade" e "Impacto Ambiental", respectivamente.

A figura 1 mostra essas semelhanças, pois apresenta as dimensões dos quatro trabalhos já referidos nessa seção, comparando suas nomenclaturas, explicando seu entendimento para esse trabalho e identificando como elas serão doravante tratadas. Importante ressaltar alguns pontos. Estabeleceu-se que para uma dimensão ser incluída na discussão do presente artigo, ela deveria ter elementos semelhantes em ao menos mais um modelo. Lamberton e Rose (2012) utilizam a dimensão "Uso do Consumidor" em seu modelo. Nenhum outro trabalho utiliza essa dimensão e, portanto, ela não foi considerada. Além disso, outra explicação na formação das dimensões desse projeto deve ser dada, no que se refere à dimensão "Prazer" de Hamari, Sjöklint e Ukkonen (2015). Ela foi aproximada pela dimensão utilidade dos demais modelos, na medida em que descreve os benefícios oriundos do consumo colaborativo.

\subsection{Tipologias da Economia Compartilhada}

Uma forma de olhar os sistemas da economia compartilhada surge a partir das tentativas de classificação propostas. Habibi et al. (2016) propuseram um modelo contínuo de classificação dos negócios advindos dos consumos que não geram propriedade para quem participa, ou acessa (Eckhardt \& Bardhi, 2016). Para Habibi et al. (2016) as organizações se posicionam entre o puro compartilhamento e a pura troca, com a possibilidade de modelos híbridos entre os dois tipos. A base desse modelo, são as características de protótipo desenvolvidas por Belk (2010). As características de Belk (2010) demonstram a diferenciação dos modelos a partir da dicotomia que eles representam em determinados elementos. A expectativa pela reciprocidade e o equilíbrio na troca, a partir de um justo cálculo pelo que 
é consumido, a partir de uma transação financeira que não gera obrigações posteriores, são características básicas de sistemas baseados em troca de mercadorias (Belk 2010). No caso de modelos de negócios baseados em puro compartilhamento, não haveria a expectativa pela reciprocidade dos envolvidos, além da propensão à criação de laços e a troca monetária não ser extremamente relevante (Habibi et al. 2016).

Figura 1 - Dimensões do Consumo Colaborativo

\begin{tabular}{|c|c|c|c|c|c|}
\hline $\begin{array}{l}\text { Lamberton e } \\
\text { Rose (2012) }\end{array}$ & $\begin{array}{l}\text { Pizzol e } \\
\text { Almeida } \\
\text { (2015) }\end{array}$ & $\begin{array}{c}\text { Hamari, } \\
\text { Sjöklint e } \\
\text { Ukkonen } \\
(2015) \\
\end{array}$ & $\begin{array}{c}\text { Mohlmann } \\
\text { (2015) }\end{array}$ & Explicação & $\begin{array}{c}\text { Identificação } \\
\text { no Artigo }\end{array}$ \\
\hline- & $\begin{array}{l}\text { Consciência } \\
\text { Sócio- } \\
\text { Ambiental }\end{array}$ & Sustentabilidade & $\begin{array}{c}\text { Impacto } \\
\text { Ambiental*** }^{* *}\end{array}$ & $\begin{array}{c}\text { Participar do consumo } \\
\text { colaborativo é associado a altos } \\
\text { níveis de preocupação com a } \\
\text { sustentabilidade } \\
\end{array}$ & $\begin{array}{c}\text { Consumo } \\
\text { Sustentável }\end{array}$ \\
\hline- & $\begin{array}{l}\text { Identidade } \\
\text { Social }\end{array}$ & Reputação & $\begin{array}{l}\text { Pertencimento } \\
\text { à Comunidade } \\
\qquad *\end{array}$ & $\begin{array}{c}\text { Ganhos percebidos no nível } \\
\text { pessoal sobre como os outros } \\
\text { percebem quem utiliza o consumo } \\
\text { colaborativo }\end{array}$ & $\begin{array}{l}\text { Identidade } \\
\text { Social }\end{array}$ \\
\hline $\begin{array}{c}\text { Controle } \\
\text { sobre o } \\
\text { produto } \\
\text { compartilhado }\end{array}$ & Confiança & - & Confiança* & $\begin{array}{c}\text { Confiança no sistema da economia } \\
\text { compartilhada e nas pessoas que } \\
\text { participam dela. }\end{array}$ & Confiança \\
\hline Familiaridade & Riscos & - & Familiaridade* & $\begin{array}{l}\text { Temor de não poder utilizar um } \\
\text { produto pela sua escassez, falta de } \\
\text { familiaridade ou falta de condições } \\
\text { de uso do produto. }\end{array}$ & Riscos \\
\hline Custos & $\begin{array}{l}\text { Economia de } \\
\text { Custos }\end{array}$ & $\begin{array}{l}\text { Benefícios } \\
\text { Econômicos }\end{array}$ & $\begin{array}{l}\text { Economia de } \\
\text { Custos* }\end{array}$ & $\begin{array}{l}\text { Percepção de economia de valores } \\
\text { monetários em transações dentro } \\
\text { da economia compartilhada. }\end{array}$ & $\begin{array}{c}\text { Economia de } \\
\text { Custos }\end{array}$ \\
\hline Utilidade & Conveniência & Prazer & Utilidade* & $\begin{array}{c}\text { Sentimentos associados ao apreço } \\
\text { e valorização dos benefícios vindos } \\
\text { da economia compartilhada }\end{array}$ & Utilidade \\
\hline
\end{tabular}

Fonte: os autores.

Outra abordagem classificatória dos modelos de negócio de economia compartilhada pode ser encontrada no trabalho de Petrini et al. (2017). Ao invés de utilizar-se de um continuum entre compartilhamento e troca como Belk (2010), os autores preocuparam-se em utilizar características próprias aos sistemas de economia compartilhada e consumo colaborativo. Importante ressaltar que se aplica aqui o entendimento de que economia compartilhada e consumo colaborativo são termos intercambiáveis e usados para discutir e compreender um mesmo fenômeno. A partir da análise da literatura, os autores identificaram 11 características. Para analisar tais características, foram selecionadas três plataformas em cada uma das 24 categorias de consumo colaborativo propostas por Botsman e Rogers (2010), totalizando um total de 72 plataformas analisadas. Quatro das 11 características permitiram o agrupamento das plataformas e levaram à proposição de três arquétipos. As quatro características foram tipo de plataforma, transação financeira, estrutura de mercado e modelo de compartilhamento. 0 tipo de plataforma pode ser interferente ou intermediadoras, envolvendo ou não a presença de transações financeiras. A estrutura de mercado relaciona-se à propriedade do bem que está sendo compartilhado, se indivíduos ou organizações. Por fim, o modelo de compartilhamento pode se dar baseado no acesso ao bem ou na transferência da propriedade deste bem. Por fim, foi possível para Petrini et al. (2017) sugerirem a existência de três grandes arquétipos dentro da economia compartilhada: negócios repaginados, novos modelos de negócios e compartilhamento como ideal (New Business Models, Redesigned Businesses e Sharing by Ideal), conforme ilustrado na figura 2.

Os negócios repaginados (Redesigned Businesses) caracterizam-se por serem plataformas B2P (business-to-peer), além de reinvenções de atividades comerciais em mercados tradicionais. São baseadas no acesso. Pelas premissas de Botsman e Rogers (2010) e de Petrini et al (2017) tal arquétipo se distancia do real modelo de compartilhamento, que busca modelos de negócios mais voltadas ao relacionamento entre pessoas (P2P). 
Os novos modelos de negócios (New Business Models) compreendem a entrada de novos players em mercados tradicionais, tal como Uber no mercado de transporte, ou Airbnb no mercado de turismo. As empresas baseiam suas ofertas tanto em acesso, quanto em transferência como modelo econômico. Seus gatilhos de consumo por parte dos usuários estão mais voltados aos elementos econômicos, sendo a sustentabilidade deixada em segundo plano (Barnes \& Mattson, 2016).

Por fim, os modelos de empresas do tipo Sharing By Ideal representam o estilo de vida colaborativo apregoado por Botsman e Rogers (2010). Seus propósitos se aproximam às definições de compartilhamento (Belk, 2007; Rudiman, 2016) e transmitem a mensagem de serem alternativas ao consumo exagerado, uma vez que não há troca financeira entre os participantes (P2P). Transcendendo a esfera dos negócios, as práticas acabam por serem percebidas com estilos de vida.

Figura 2 - Tipologia de Economia Compartilhada

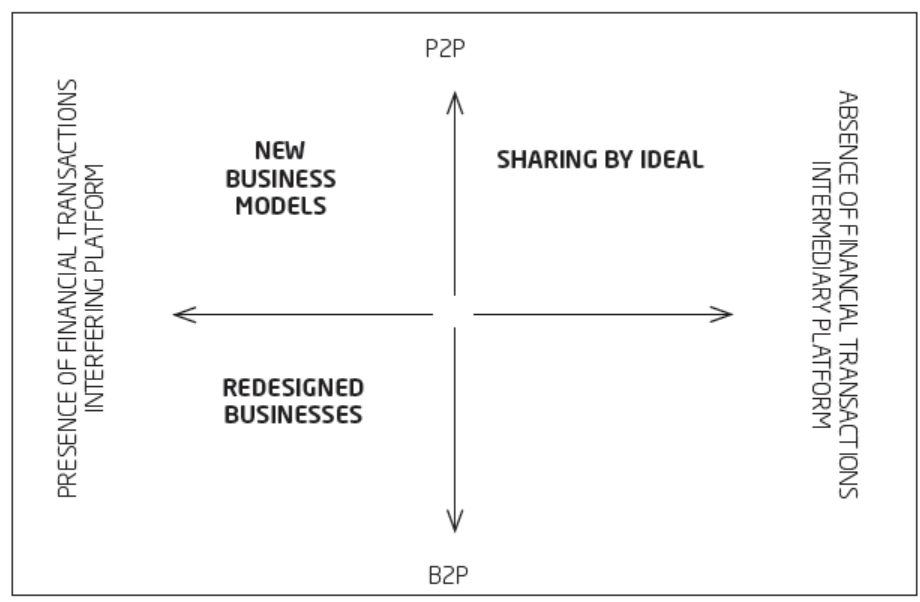

Fonte: Petrini, Freitas \& Silveira (2017).

\section{Discussão}

Nas próximas linhas se aprofunda a discussão sobre como as dimensões do consumo colaborativo são construídas mentalmente - se mais abstrata ou concretamente - e como isso pode ter efeito nos tipos diferentes de negócios, em especial naqueles caracterizados como "Novos modelos de negócios" e "Compartilhamento como ideal" de Petrini et al (2017). Optou-se por descartar os modelos de "negócios repaginados" dadas as características que os formam, e por estarem ligeiramente mais afastados das premissas da economia compartilhada (Petrini et al., 2017; Botsman \& Rogers, 2010). Os próprios autores declaram que este arquétipo é o que mais se distancia da economia compartilhada considerando os motivadores para sua existência. Adicionalmente, dado nosso interesse em compreender a economia compartilhada à luz das representações mentais dos indivíduos, no arquétipo "negócios repaginados" a estrutura de mercado é B2P, ou seja, o bem é de propriedade de uma organização (e não de indivíduos) e compartilhado por diferentes indivíduos. Iniciamos nossa discussão apresentando os argumentos acerca das dimensões da economia compartilhada e suas potenciais representações mentais.

Imagina-se que a escolha por participar da economia compartilhada poderia ser explicada, em parte, por uma atenção maior a questões ambientais e de sustentabilidade, otimizando as consequências sociais, econômicas e ambientais do consumo, olhando para as gerações do presente e do futuro (Luchs, Naylor, Rose, Catlin \& Gau 2011). Entretanto, a literatura já demonstrou que esse motivo não parece ser tão importante para os consumidores da economia compartilhada (Mohlmann, 2015; Barnes \& Mattson, 2016). Mesmo assim, a preocupação com um consumo sustentável ainda se justifica na explicação do fenômeno da economia compartilhada. Tanto persiste, que é considerada uma de suas dimensões (Lamberton \& Rose, 2012; Hamari et al 2015). Entretanto, uma dimensão que parece não ter um efeito tão forte no comportamento de participar, mas sim na atitude sobre o fenômeno (Hamari et al 2015). Uma explicação para isso pode estar na forma como essa dimensão é construída na mente do consumidor. Há a constatação na literatura que as escolhas sustentáveis geralmente se referem a eventos de interpretação mental mais abstrata (Rabinovich, Morton, Postmes \& Verplanken, 2009; Spence, Poortinga \& Pidgeon 2012; van Dam \& van Trijp, 2013). Portanto, as escolhas e resultados sustentáveis são construídos em termos de vontade, enquanto o comportamento habitual seria construído em baixos níveis de interpretação. Dessa forma, o apelo de sustentabilidade ou de diminuição 
de impactos ambientais, muitas vezes relacionado com o consumo colaborativo (Belk, 2010; Martin, 2016; Habibi et al., 2017), parece não ter o efeito de levar o consumidor a tomar a ação de acessar esse modelo econômico sem propriedade, mas impactar apenas a sua atitude.

A dimensão confiança não está descrita no modelo de Hamari et al. (2015), mas está presente nos trabalhos de Pizzol e Almeida (2015), Mohlmann (2015) e também de Lamberton e Rose (2012). As pessoas, por muitas vezes, temem participar da economia compartilhada por não ter confiança na outra parte que está envolvida. Ao se analisar as premissas de Botman e Rogers (2011) - massa crítica, capacidade ociosa, crença no bem comum e confiança entre desconhecidos -, percebe-se que esse elemento é essencial para o funcionamento do consumo colaborativo. Do ponto de vista da CLT, é estabelecido que pessoas menos familiares, ou menos similares, são consideradas pessoas socialmente mais distantes, sendo representadas de forma abstrata (Trope \& Liberman, 2003; Trope, et al., 2007). Dessa forma, o fato de as pessoas não se engajarem de forma tão semelhante às suas atitudes em relação ao consumo colaborativo pode ser justificada pela distância psicológica que existe na construção da confiança por parte dos pares que estão compartilhando alguma coisa. 0 consumo colaborativo tem em sua essência a troca e o compartilhamento entre pares. Isso só é possível quando existe a confiança de uma parte em outra. A confiança surge de sentimentos que percebemos nos outros ou em nossos julgamentos (Tsai \& Mcgill, 2011). Quanto mais houver confiança na outra parte da transação, maior será a percepção de proximidade percebida em relação ao outro, ou seja, menos abstrata estará a situação. Esse incremento na confiança tem potencial para elevar a chance da adoção de um comportamento dentro do consumo colaborativo.

Outra dimensão importante, e que também não consta no modelo de Hamari et al. (2015), é a aquela associada aos riscos. Lamberton e Rose (2012) já haviam alertado para a importância desse elemento, ao falar da incerteza e da escassez e de como ambas se relacionam com o acesso à economia compartilhada. Mueller, Wakslak e Krishnan (2014) comentam que a exposição a estímulos novos e não familiares tende a ativar processamentos abstratos nos indivíduos, pois uma perspectiva mais ampla acaba por preparar melhor para a compreensão da informação (Förster, Liberman \& Shapira, 2009; Förster, Marguc \& Gillebaart, 2010). Além disso, pessoas em níveis de interpretação mais próximos estão em um estágio onde a familiaridade com o produto em análise é importante. Retomando os conceitos iniciais de Trope e Liberman (2003), uma oferta, seja produto ou serviço, possui características primárias e secundárias. As primeiras estão voltadas ao fim do uso de determinado produto, enquanto as últimas voltam-se ao meio, ou seja, ao processo (Liberman \& Trope, 2014). Diante, portanto, de uma nova proposta de consumo, em que não se conhecem com clareza os meios que farão com que o consumidor atinja o resultado esperado para aquele consumo, a familiaridade tende a diminuir e os riscos, a aumentar. Familiaridade menor ativa processamentos abstratos (Mueller et al., 2014). A ideia da familiaridade do uso foi trazida por Pizzol e Almeida (2015) na sua mensuração do construto do consumo colaborativo, e também por Mohlmann (2015).

Um aspecto importante e presente nos estudos quantitativos de Lamberton e Rose (2012), Hamari et al. (2015), Pizzol e Almeida (2015) e Mohlmann (2015) é referente à economia de custos associada à economia compartilhada. Hamari, et al. (2015) colocam esse elemento financeiro significativamente relacionado com o comportamento. Ou seja, entende-se que, para adotar o consumo colaborativo, a variável preço é importante. Barnes e Mattsson (2016) já demonstraram a importância dessa variável para a decisão de participar na economia compartilhada. Seguindo a linha de Bornemann e Homburg (2011), quando há a percepção de sacrifícios em uma operação, o consumidor tende a construir o preço em baixo nível. Dada a mudança de cultura de consumo inerente ao consumo colaborativo, é possível especular que migrar para um novo sistema implique em novos empecilhos, que ativem construções mentais de mais baixo nível. Além disso, um maior número de pesquisas relatadas na descrição do subcampo monetário da pesquisa em CLT, apontou o preço como sendo um elemento de construção em baixos níveis (Sordi, 2017).

Faz-se relevante comentar um ponto importante da economia compartilhada, normalmente mencionado como vital à sua adoção e que pareceu negligenciado nos modelos analisados: o papel da propriedade dentro do consumo colaborativo. Os sentimentos de apego à posse são ditos por Belk (2010) como limitadores do compartilhamento, pois afetam a vontade de compartilhar, uma vez que existe uma forte ligação emocional entre dono e produto. 0 estudo de Irmak et al. (2013) mostra que as pessoas que detêm a posse de um produto constroem seus julgamentos em níveis de interpretação mais altos. É possível compreender disso que umas das principais barreiras descritas pelos teóricos (Belk, 
2010; Pizzol \& Almeida, 2015) para a economia compartilhada acontece pela construção mental dos indivíduos que possuem a propriedade do produto. Para que haja a adoção, ou seja, para que se crie o comportamento de uso, é necessária uma modificação na interpretação mental, passando de uma construção de alto nível para níveis mais baixos, focando-se em pontos mais concretos, como na facilidade e familiaridade de uso, na economia de custos e na confiança de quem participa. A questão da propriedade, auxilia a entender os motivos da dimensão da utilidade de Lamberton e Rose (2012) e Mohlmann (2015) ser construída em baixo nível de interpretação, pois está fortemente relacionado ao comportamento que será tomado ao participar do consumo colaborativo. 0 sentido dessas dimensões similares é a valorização dos benefícios da economia compartilhada. Considerando a ausência da propriedade de um produto como um dos elementos centrais do consumo colaborativo, é possível especular que a propriedade estimula níveis mais altos (Irmak et al., 2013) e a utilidade mais baixos da interpretação mental.

A dimensão de identidade social possui peculiaridades em sua forma de representação. Eyal, Sagristano, Trope, Liberman e Chaiken (2009) dizem que as pessoas pensam sobre si mesmas e suas ações, valores, ideologias e princípios de forma abstrata. Como as dimensões de reputação, de Hamari et al. (2015), e de identidade social, de Pizzol e Almeida (2015), correspondem a essa visão de si mesmo que o indivíduo tem, a partir de seus valores, essa seria uma construção em nível mais alto. As características de protótipo de Belk (2010) são também importantes para compreender melhor o que está sendo dito. Para o autor, a interação e ligação social que resultam em estruturas de compartilhamento são essenciais para esses modelos de negócio. Ao estar vinculado a tais modelos, o indivíduo pode estar sujeito a gerar mais pensamentos abstratos de si mesmos, graças a essa construção de suas ações em alto nível de interpretação mental (Eyal et al., 2009).

Importante mencionar neste ponto, que o trabalho de Hamari et al. (2015) testou os impactos das suas dimensões (sustentabilidade, aproveitamento, reputação e benefícios econômicos) na atitude e no comportamento de adoção dos indivíduos. Os autores identificaram impactos diferentes das dimensões nas variáveis dependentes. Sustentabilidade, por exemplo, impactou mais a atitude do que o comportamento. Assim como a dimensão econômica impacta mais no comportamento do que na atitude. Nesse sentido, surge o gap já relatado neste projeto. Ressalva-se que as dimensões de confiança e familiaridade não estavam presentes nas análises de Hamari et al. (2015), mas surgem nas demais (Lamberton \& Rose, 2012; Molmann, 2015; Pizzol \& Almeida, 2015). O conceito e a relação entre atitude e comportamento deriva da Teoria do Comportamento Planejado de Ajzen (1991). A atitude reflete o nível com que um indivíduo avalia positiva ou negativamente um fenômeno (Ajzen, 2001). As atitudes de um indivíduo convergem em potenciais antecedentes para seu comportamento (Ajzen, 1991; Liñán \& Chen, 2009). Nesse sentido, Hamari et al. (2015) sustentam que a relação entre atitude e comportamento no consumo colaborativo apresenta uma discrepância importante se comparado a outros estudos que relacionam os dois construtos. Os autores sustentam que a relação existe, mas em níveis mais modestos.

Uma possível explicação para a discrepância observada, surge a partir dos diferentes efeitos das dimensões sobre a atitude e o comportamento. E é possível relacionar essa situação com a forma com que as dimensões são construídas mentalmente pelos indivíduos. Conforme já discutido, as dimensões do consumo colaborativo teriam suas interpretações ora de alto nível e abstrata, ora de baixo nível e concreta. Essa situação (interpretações mentais de cada dimensão) influencia o comportamento do consumidor. Três das seis dimensões (Consumo Sustentável, Riscos e Identidade Social) tem uma relação potencial com níveis mais altos, e outras três (Economia de Custos, Utilidade e Confiança) de baixo nível. 0 que se propõe, portanto, é que as dimensões que são construídas em alto nível impactarão com mais intensidade a atitude. Em contrapartida, o comportamento será afetado mais fortemente pelas dimensões construídas em baixo nível. Esse argumento surge a partir das observações e levantamentos feitos por Arts et al. (2011) na busca por identificar qual o efeito dos níveis de interpretação mental na adoção de inovações.

Além das dimensões do consumo colaborativo terem a possibilidade de sofrer influência dos seus níveis de construção, os próprios modelos negócios podem ser construídos em níveis distintos. Trope e Liberman (2003) sustentam que a construção mental, de forma geral, são representações que os indivíduos criam em suas mentes sobre produtos, objetos, pessoas, lugares, ou seja, para qualquer tipo de avaliação a ser feita. Nesse sentido, cabe especular que a mesma representação pode ser feita sobre empresas que propõem negócios no contexto do consumo colaborativo. Tomando como base os 
trabalhos de Belk (2010), Habibi et al. (2016), e Petrini et al. (2017), a interpretação mental dos tipos de modelos de negócio da economia compartilhada também podem variar de acordo com os níveis de construção dos indivíduos. Ao analisar as características de protótipo de Belk (2010), percebe-se que aquelas associadas a negócios do tipo Sharing by Ideal (não-recícproco, ligações sociais, irrelevância do dinheiro, relacionamento, pessoal, inalienável, entre outras) podem ser associadas a condições de abstração, ou mesmo questões fim do negócio. Já nos modelos batizados como "New Business Models", as características de Belk (2010) (troca balanceada, monetária, inspeção, cálculo, entre outras) podem ser construídas em representações concretas. A percepção dessas características poderia, também, ser estendida ao modelo de negócio, fazendo com que seja possível avaliar e perceber o nível de abstração em que os negócios colaborativos são formados. Essa situação, poderia auxiliar a explicar o gap de atitude-comportamento de Hamari et al. (2015).

Hamari et al. (2015) testaram o seu modelo utilizando o consumo colaborativo de maneira geral. Suas questões, em nenhum momento, focaram-se em um modelo de negócios já existentes, como compartilhamento de carros ou bicicleta (Lamberton \& Rose, 2012; Pizzol \& Almeida, 2015). Portanto, o gap encontrado, de certa forma, não foi contextualizado. Com o avanço da pesquisa no campo da economia compartilhada e a construção desses modelos de classificação e tipologias (Habibi et al., 2016; Petrini et al., 2017), faz sentido ampliar a observação das dimensões e do gap entre atitude e comportamento, cruzando-as pelos tipos de negócios, no sentido de verificar quais dimensões impactam quais tipos de negócios mais fortemente.

É possível especular que o tipo de negócio proposto possa reduzir o distanciamento da atitude e do comportamento. Habibi et al. (2016) desenvolveram uma escala para medir o índice de compartilhamento (sharing score) que os modelos de negócios desse novo ambiente econômico apresentariam. Valores mais altos, posicionariam os negócios mais à esquerda dentro do continuum dentro da lógica de puro compartilhamento, enquanto valores mais baixos demonstravam uma associação maior à modelos de pura troca (HABIBI et al., 2016). Nos estudos realizados, por exemplo, a plataforma Couchsurfing.com, que é uma rede de hospitalidade gratuita (Molz, 2013), teve um índice de compartilhamento mais elevado. Na classificação de Petrini et al (2017) a plataforma foi caracterizada como um negócio de tipo "Sharing by Ideal", onde não há presença de transação financeira, mas sim uma preocupação com valores envolvidas no consumo. Plataformas como o Airbnb foram posicionadas como modelos intermediários por Habibi et al (2016). Para Petrini et al (2017) tais modelos se encaixam como "New Business Models" pelas suas características de serem peer-to-peer e envolverem transações financeiras. Por fim, modelos como Zipcar foram caracterizados como negócios de pura troca por Habibi et al (2016) e como sendo parte dos negócios repaginados de Petrini et al (2017). Em modelos como esses, tudo funciona de forma balanceada, com a lógica perfeita dos sistemas de troca. Isso reforça a opção de descarte desses modelos na discussão que se desenvolve sobre os níveis de interpretação dos negócios no contexto da economia compartilhada.

Figura 3 - Esquema teórico proposto

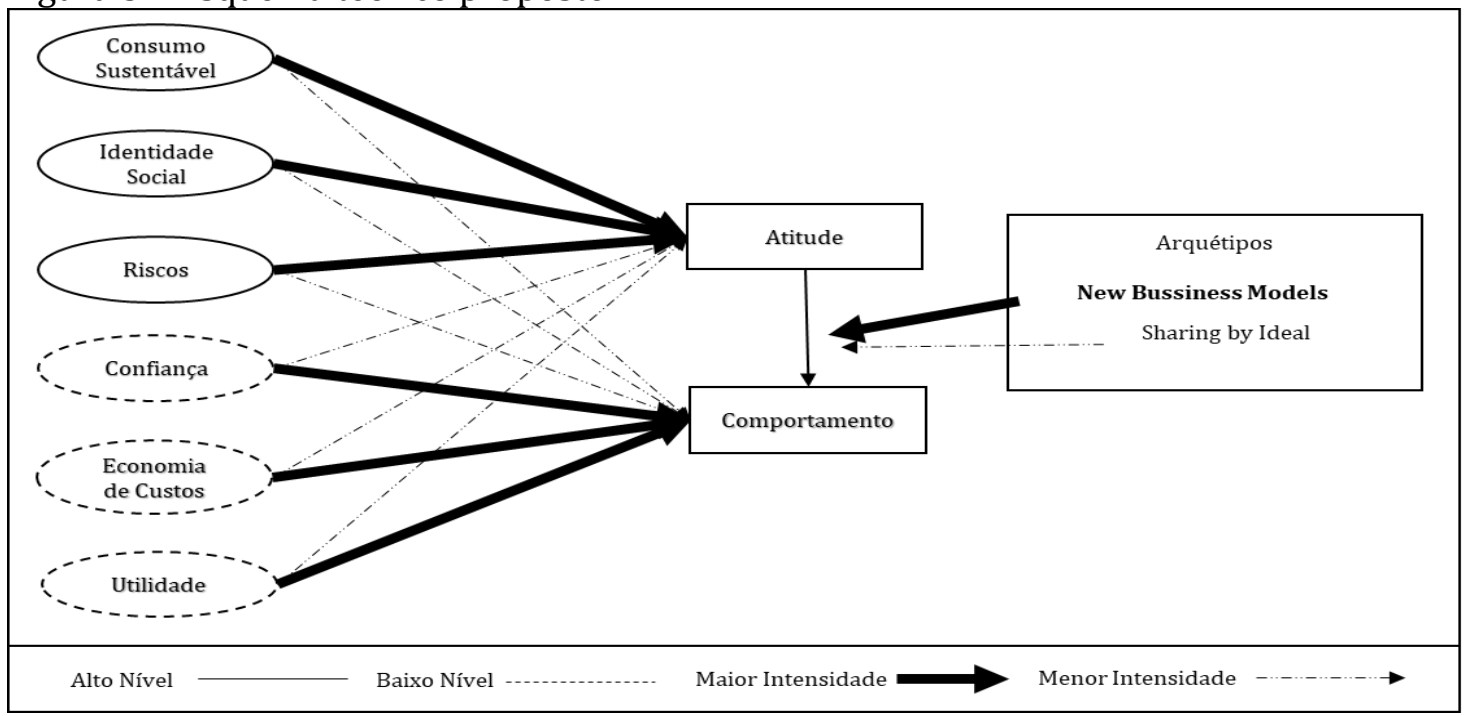

Fonte: os autores. 
O que se pode especular, a partir do exposto, é que o tipo de negócio pode surgir como um atenuante do gap. Para os "New Business Models", que possuem as características de troca monetária entre pares, implicando uma reciprocidade (Belk, 2010; Petrini et al, 2017), a atitude e o comportamento teriam uma relação mais intensa, pois o consumidor conseguiria perceber mais facilmente como a transação aconteceria e se sentiria mais interessado em participar desse tipo de consumo. Já para modelos do tipo "Sharing by Ideal", que são desenvolvidos à luz de laços sociais nãorecíprocos e que o dinheiro se torna irrelevante (BELK, 2010; Petrini et al. 2017), a diferença seria maior. Essas ideias são esquematizadas na figura 3.

\section{Conclusões}

O objetivo do presente trabalho era desenvolver, através de uma abordagem teórica, uma discussão sobre a participação no fenômeno da economia compartilhada. Em nenhum momento houve pretensão de pesquisar os elementos ou atributos mais importantes para os consumidores, algo já bastante discutido na literatura (Piscicelli et al, 2015; Lawson et al, 2016; Tussyadiah, 2016; Ert, Fleischer \& Magen, 2016; Barnes \& Mattsson, 2016). A aspiração do presente foi apresentar uma explicação para os motivos de tais elementos serem importantes - ou não - para a participação na economia compartilhada. Ao sugerir que a dimensão "consumo sustentável" seja representada mais abstratamente, e com menos impacto no comportamento e mais na atitude, o presente artigo encontra eco nos demais textos que dizem que o tema de sustentabilidade não é preponderante à participação na economia compartilhada (Barnes \& Mattsson, 2016). Da mesma forma, ao citar o possível impacto do preço no comportamento, dada a sua natureza de ser representado mentalmente de forma concreta, esse ensaio não entra em contradição com os textos de Lawson et al. (2016), Tussyadiah (2016) e Böcker e Mellen, (2017). Antes disso, os corrobora, tentando explicar o porquê de tais resultados encontrados.

E reside nesses fatos uma das contribuições do presente trabalho à literatura de economia compartilhada. 0 interesse está em compreender como a Construal Level Theory, através das representações mentais, pode ajudar a explicar do ponto de vista cognitivo a participação na economia compartilhada. Ao comentar isso, é importante ressaltar que o artigo também contribui à literatura de CLT, pois é mais uma nova aplicação dos seus conceitos, na busca por explicar mais um fenômeno contemporâneo, como o consumo colaborativo. 0 volume de textos que buscam relacionar os conceitos da CLT com a economia compartilhada ainda é restrito (Sordi, Perin, Petrini \& Sampaio 2018; Schereiner, Pick \& Kenning, 2018). Por fim, uma última contribuição do presente estudo e que pode ser destacada é o fato de utilizar-se de uma tipologia para avançar no possível gap entre atitude e comportamento descrito por Hamari et al, (2015). Ao propor que o gap pode ter intensidade variada pelo tipo de negócio, respaldado pelos arquétipos de Petrini et al (2017), o artigo tenta expandir o modelo de Hamari et al, (2015) e trazer explicações alternativas para sua ocorrência.

0 trabalho deixa, também, novos direcionamentos para pesquisas futuras. 0 primeiro deles é o próprio teste das relações e proposições ora levantadas. Além disso, é possível tentar observar os efeitos das distâncias psicológicas - social, temporal, hipotética e espacial - de forma isolada na participação na economia compartilhada. Por fim, é possível explorar também de forma mais detalhada cada uma das dimensões apresentadas no texto e suas relações e efeitos nos consumidores do contexto em questão. De certa forma, os elementos propostos aqui convergem para um aumento dos estudos sobre os fatores motivacionais que afetem as intenções e comportamentos dos consumidores diante da economia compartilhada (Lamberton \& Rose, 2012; Hamari et al, 2015).

\section{Referências}

Ajzen, I. (1991). The theory of planned behavior. Organizational behavior and human decision processes, 50(2), 179-211.

Ajzen, I. (2001). Nature and operation of attitudes. Annual review of psychology, 52(1), 27-58.

Arts, J. W., Frambach, R. T., \& Bijmolt, T. H. (2011). Generalizations on consumer innovation adoption: A meta-analysis on drivers of intention and behavior. International Journal of Research in Marketing, 28(2), 134-144.

Bardhi, F., \& Eckhardt, G. M. (2012). Access-based consumption: The case of car sharing. Journal of consumer research, 39(4), 881-898.

Barnes, S. J., \& Mattsson, J. (2016). Understanding current and future issues in collaborative consumption: A four-stage Delphi study. Technological Forecasting and Social Change, 104, 200-211. 
Belk, R. (2007). Why not share rather than own?. The Annals of the American Academy of Political and Social Science, 611(1), 126-140.

Belk, R. (2010). Sharing. Journal of consumer research, 36(5), 715-734.

Belk, R. (2014). You are what you can access: Sharing and collaborative consumption online. Journal of Business Research, 67(8), 1595-1600.

Böcker, L., \& Meelen, T. (2017). Sharing for people, planet or profit? Analysing motivations for intended sharing economy participation. Environmental Innovation and Societal Transitions, 23, 28-39.

Bornemann, T., \& Homburg, C. (2011). Psychological distance and the dual role of price. Journal of Consumer Research, 38(3), 490-504.

Botsman, R., \& Rogers, R. (2009). O que é meu é seu: como o consumo colaborativo vai mudar o nosso mundo. Bookman Editora.

Branco, A. C., \& Nunes, J. M. G. (2018). Impactos das plataformas P2P na economia do compartilhamento. PMKT - Revista Brasileira de Pesquisas de Marketing, Opinião e Mídia, 11(2), 222-235.

Cheema, A., \& Patrick, V. M. (2008). Anytime versus only: mind-sets moderate the effect of expansive versus restrictive frames on promotion evaluation. Journal of Marketing Research, 45(4), 462-472.

Chen, R., Xu, X., \& Shen, H. (2017). Go beyond just paying: Effects of payment method on level of construal. Journal of Consumer Psychology, 27(2), 207-217.

Chen, Y. (2009). Possession and access: Consumer desires and value perceptions regarding contemporary art collection and exhibit visits. Journal of Consumer Research, 35(6), 925-940.

Cho, E. K., Khan, U., \& Dhar, R. (2013). Comparing apples to apples or apples to oranges: The role of mental representation in choice difficulty. Journal of Marketing Research, 50(4), 505-516.

Dubois, E., Schor, J., \& Carfagna, L. (2014). Connected consumption: a sharing economy takes hold. Rotman Management, 50-55.

Eckhardt, G. M., \& Bardhi, F. (2016). The relationship between access practices and economic systems. Journal of the Association for Consumer Research, 1(2), 210-225.

Ert, E., Fleischer, A., \& Magen, N. (2016). Trust and reputation in the sharing economy: The role of personal photos in Airbnb. Tourism Management, 55, 62-73.

Eyal, T., Sagristano, M. D., Trope, Y., Liberman, N., \& Chaiken, S. (2009). When values matter: Expressing values in behavioral intentions for the near vs. distant future. Journal of Experimental Social Psychology, 45(1), 35-43.

Felson, M., \& Spaeth, J. L. (1978). Community structure and collaborative consumption: A routine activity approach. American behavioral scientist, 21(4), 614-624.

Förster, J., Liberman, N., \& Shapira, O. (2009). Preparing for novel versus familiar events: Shifts in global and local processing. Journal of experimental psychology: general, 138(3), 383.

Förster, J., Marguc, J., \& Gillebaart, M. (2010). Novelty categorization theory. Social and Personality Psychology Compass, 4(9), 736-755.

Gansky, L. (2010). The mesh: Why the future of business is sharing. Penguin.

Godelier, M. (2012). The metamorphoses of kinship. Verso Books.

Gollnhofer, J. F., Hellwig, K., \& Morhart, F. (2016). Fair is good, but what is fair? Negotiations of distributive justice in an emerging nonmonetary sharing model. Journal of the Association for Consumer Research, 1(2), 226-245.

Goodman, J. K., \& Malkoc, S. A. (2012). Choosing here and now versus there and later: The moderating role of psychological distance on assortment size preferences. Journal of Consumer Research, 39(4), 751768.

Habibi, M. R., Davidson, A., \& Laroche, M. (2017). What managers should know about the sharing economy. Business Horizons, 60(1), 113-121.

Habibi, M. R., Kim, A., \& Laroche, M. (2016). From sharing to exchange: An extended framework of dual modes of collaborative nonownership consumption. Journal of the Association for Consumer Research, 1(2), 277-294.

Hamari, J., Sjöklint, M., \& Ukkonen, A. (2016). The sharing economy: Why people participate in collaborative consumption. Journal of the Association for Information Science and Technology, 67(9), 2047-2059.

Irmak, C., Wakslak, C. J., \& Trope, Y. (2013). Selling the forest, buying the trees: The effect of construal level on seller-buyer price discrepancy. Journal of Consumer Research, 40(2), 284-297. 
Kaplan, A. M., \& Haenlein, M. (2010). Users of the world, unite! The challenges and opportunities of Social Media. Business horizons, 53(1), 59-68.

Lamberton, C. P., \& Diehl, K. (2013). Retail choice architecture: The effects of benefit-and attribute-based assortment organization on consumer perceptions and choice. Journal of Consumer Research, 40(3), 393-411.

Lamberton, C. P., \& Rose, R. L. (2012). When is ours better than mine? A framework for understanding and altering participation in commercial sharing systems. Journal of Marketing, 76(4), 109-125.

Lawson, S. J., Gleim, M. R., Perren, R., \& Hwang, J. (2016). Freedom from ownership: An exploration of access-based consumption. Journal of Business Research, 69(8), 2615-2623.

Liberman, N., \& Trope, Y. (2014). Traversing psychological distance. Trends in Cognitive Sciences, 18(7), 364-369.

Liñán, F., \& Chen, Y. W. (2009). Development and Cross-Cultural application of a specific instrument to measure entrepreneurial intentions. Entrepreneurship theory and practice, 33(3), 593-617.

Luchs, M., Naylor, R. W., Rose, R. L., Catlin, J. R., Gau, R., Kapitan, S., ... \& Subrahmanyan, S. (2011). Toward a sustainable marketplace: Expanding options and benefits for consumers. Journal of Research for Consumers, (19), 1.

Markus, K., \& Orsi, D. F. Um estudo do consumo colaborativo no Brasil e nos Estados Unidos da América. Revista Organizações em Contexto, 12(24), 117-129.

Martin, C. J. (2016). The sharing economy: A pathway to sustainability or a nightmarish form of neoliberal capitalism?. Ecological Economics, 121, 149-159.

Möhlmann, M. (2015). Collaborative consumption: determinants of satisfaction and the likelihood of using a sharing economy option again. Journal of Consumer Behaviour, 14(3), 193-207.

Molz, J. G. (2013). Social networking technologies and the moral economy of alternative tourism: The case of couchsurfing. org. Annals of tourism research, 43, 210-230.

Mueller, J. S., Wakslak, C. J., \& Krishnan, V. (2014). Construing creativity: The how and why of recognizing creative ideas. Journal of Experimental Social Psychology, 51, 81-87.

Petrini, M., Freitas, c. S. D., \& Silveira, l. M. D. (2017). A proposal for a typology of sharing economy. Revista de Administração Mackenzie, 18(5), 39-62.

Piran, F. A. S., Neves, C. T., Sordi, J. D. \& Nunes, F. L. (2018). A economia compartilhada e a percepção de seus efeitos por parte dos estudantes de uma instituição de ensino superior. Consumer Behavior Review, 2(Special Edition), 69-80.

Piscicelli, L., Cooper, T., \& Fisher, T. (2015). The role of values in collaborative consumption: insights from a product-service system for lending and borrowing in the UK. Journal of Cleaner Production, 97, 21-29.

Pizzol, H., \& Almeida, S. (2015). Compreendendo o compartilhamento de bens: proposição de uma escala para mensuração do construto consumo colaborativo. Anais do Encontro da ANPAD, Belo Horizonte, MG, Brasil, 39. Recuperado de: http://www.anpad.org.br/ anpad/abrir pdf.php?e=MjAyOTc=

Price, L. L., \& Belk, R. W. (2016). Consumer ownership and sharing: Introduction to the issue. Journal of the Association for Consumer Research, 1(2), 193-197.

Rabinovich, A., Morton, T. A., Postmes, T., \& Verplanken, B. (2009). Think global, act local: The effect of goal and mindset specificity on willingness to donate to an environmental organization. Journal of Environmental Psychology, 29(4), 391-399.

Reyt, J. N., \& Wiesenfeld, B. M. (2015). Seeing the forest for the trees: Exploratory learning, mobile technology, and knowledge workers' role integration behaviors. Academy of Management Journal, 58(3), 739-762.

Richins, M. L. (1994). Special possessions and the expression of material values. Journal of consumer research, 21(3), 522-533.

Rifkin, J. (2001). The age of access: The new culture of hypercapitalism. Penguin.

Rudmin, F. (2016). The consumer science of sharing: A discussant's observations. Journal of the Association for Consumer Research, 1(2), 198-209.

Schor, J. (2016). DEBATING THE SHARING ECONOMY.Journal of Self-Governance \& Management Economics, 4(3).

Schreiner, N., Pick, D., \& Kenning, P. To share or not to share? Explaining willingness to share in the context of social distance. Journal of Consumer Behaviour, 2018, 1-13. 
Schwartz, S. H., Cieciuch, J., Vecchione, M., Davidov, E., Fischer, R., Beierlein, C., ... \& Dirilen-Gumus, O. (2012). Refining the theory of basic individual values.Journal of personality and social psychology, 103(4), 663.

Schwartz, S. H., Melech, G., Lehmann, A., Burgess, S., Harris, M., \& Owens, V. (2001). Extending the crosscultural validity of the theory of basic human values with a different method of measurement. Journal of cross-cultural psychology, 32(5), 519-542.

Sordi J. D. (2017). Construal level theory e comportamento do consumidor: reconhecimento do campo e novas oportunidades de pesquisa. Anais do Seminário de Administração, São Paulo, SP, 20. Recuperado de http://login.semead.com.br/20semead/arquivos/1156.pdf

Sordi, J. D., Perin, M. G., Petrini, M. D. C., \& Sampaio, C. H. (2018). Construal level and collaborative consumption: An exploratory approach. International Journal of Consumer Studies, 42(2), 264-273.

Spence, A., Poortinga, W., \& Pidgeon, N. (2012). The psychological distance of climate change. Risk analysis, 32(6), 957-972.

Sun, J., Supangkat, H., \& Balasubramanian, S. (2016). Peer-to-peer sharing of private goods: Sellers' response and consumers' benefits. Journal of the Association for Consumer Research, 1(2), 262-276.

Trope, Y., \& Liberman, N. (2003). Temporal construal. Psychological review, 110(3), 403-421.

Trope, Y., \& Liberman, N. (2010). Construal-level theory of psychological distance. Psychological review, 117(2), 440-463.

Trope, Y., Liberman, N., \& Wakslak, C. (2007). Construal levels and psychological distance: Effects on representation, prediction, evaluation, and behavior. Journal of consumer psychology, 17(2), 83-95.

Tsai, C. I., \& McGill, A. L. (2010). No pain, no gain? How fluency and construal level affect consumer confidence. Journal of Consumer Research, 37(5), 807-821.

Tussyadiah, I. P. (2016). Factors of satisfaction and intention to use peer-to-peer accommodation. International Journal of Hospitality Management, 55, 70-80.

van Dam, Y. K., \& van Trijp, H. C. (2013). Relevant or determinant: Importance in certified sustainable food consumption. Food quality and preference, 30(2), 93-101.

Wiesenfeld, B. M., Reyt, J. N., Brockner, J., \& Trope, Y. (2017). Construal level theory in organizational research. Annual Review of Organizational Psychology and Organizational Behavior, 4, 367-400.

Wolcott, R. (2014). Kellogg innovation network executive forum. Kellogg School of Management: Evaston, USA.

Yan, D., \& Sengupta, J. (2011). Effects of construal level on the price-quality relationship. Journal of Consumer Research, 38(2), 376-389.

Zhao, M., \& Xie, J. (2011). Effects of social and temporal distance on consumers' responses to peer recommendations. Journal of Marketing Research, 48(3), 486-496. 